RECyT

Año 22 / No 33 / 2020 / 94-99

\title{
Estudio preliminar para optimizar la extracción de compuestos fenólicos bioactivos de quinoa (Chenopodium quinoa Willd)
}

\section{Preliminary study to optimize the extraction of bioactive phenolic compounds of quinoa (Chenopodium quinoa Willd)}

\author{
Julia Luisetti1 ${ }^{1}{ }^{*}$, Héctor Lucero ${ }^{1}$, María C. Ciappini ${ }^{1}$ \\ 1- CIDTA (Centro de Investigación y Desarrollo en Tecnología de los Alimentos). Facultad Regional \\ Rosario. Universidad Tecnológica Nacional. Zeballos 1341, Rosario. Santa Fe, Argentina. \\ *E-mail: julialuisetti@yahoo.com.ar \\ Recibido el 08 de octubre de 2019, Aprobado el 27 de febrero de 2020.
}

\section{Resumen}

\begin{abstract}
Dentro de los antioxidantes naturales encontrados en la quinoa (Chenopodium quinoa Willd) se destaca la importancia de los compuestos fenólicos, cuya aplicación en la formulación de potenciales alimentos funcionales, por sus efectos beneficiosos para la salud, hace interesante su extracción de manera selectiva. Se propone optimizar los parámetros del proceso de extracción de los compuestos fenólicos, sobre la base de un diseño experimental de 3 variables a 3 niveles. Los parámetros fueron temperatura de secado, relación Liquido/Sólido (L/S) y concentración de etanol en el solvente; sus niveles fueron, respectivamente, 40,60 y 80 $\mathrm{C}$; 20:1, 30:1 y 40:1 y $30,50,70 \%$ v/v. La cuantificación de los polifenoles se realizó según el método de Folin-Ciocalteu, expresando los resultados como mg de ácido gálico equivalente (AGE)/100 g de quinoa (en base seca). Se obtuvieron resultados entre 179 y $229 \mathrm{mg}$ de AGE /100 g de quinoa. Las predicciones del modelo se correlacionaron con los valores experimentales en un $93,62 \%$. La relación L/S óptima fue $31: 1$ y la temperatura óptima fue de $54^{\circ} \mathrm{C}$. Para la concentración de etanol en el solvente, la variable de mayor influencia en la extracción de compuestos fenólicos, el óptimo fue de $39 \%$.
\end{abstract}

Palabras clave: Quinoa; Fenoles; Optimización; Extracción hidroalcohólica; Antioxidantes.

\section{Abstract}

Among the natural antioxidants found in quinoa (Chenopodium quinoa Willd), it stands out the importance of phenolic compounds, whose application in the formulation of potential functional foods, due to their beneficial health effects, it makes their selective extraction interesting. It is proposed to optimize the parameters of the extraction process of phenolic compounds, based on an experimental design of 3 variables at 3 levels. The parameters were: drying temperature, Liquid/Solid ratio (L/S) and concentration of ethanol in the solvent; their levels were, respectively, 40, 60 and $80^{\circ} \mathrm{C} ; 20: 1,30: 1$ and $40: 1$ and $30,50,70 \% \mathrm{~V} / \mathrm{v}$. The polyphenols quantification was carried out according to the Folin-Ciocalteu method, expressing the results as $\mathrm{mg}$ of gallic acid equivalents (GAE)/100 g of quinoa (dry basis). The obtained results were between 179 and $227 \mathrm{mg}$ of AGE/100 g of quinoa. The model predictions were correlated with experimental values at $93.62 \%$. The optimum L / S ratio was $31: 1$ and the optimum temperature was $54^{\circ} \mathrm{C}$. For the ethanol concentration in the solvent, the variable with the greatest influence on the extraction of phenolic compounds, the optimum was $39 \%$.

Keywords: Quinoa; Phenols; Optimization; Hydroalcoholic extraction; Antioxidants.

\section{Introducción}

Existe una tendencia a nivel mundial en diseñar nuevos productos alimenticios utilizando granos ancestrales, ya sea suplementando o sustituyendo granos de cereales tradicionales. Por otro lado, en los últimos años aumentó el interés en especies vegetales no gramíneas, consideradas agronómicamente como pseudocereales, cuyas semillas pueden ser utilizadas de manera similar a los granos de cereales.

La quinoa es una planta anual originaria de la región andina, cultivada desde Colombia hasta Argentina y Chile, siendo los principales productores Bolivia, Perú y Ecuador $[1,18]$. Su uso como alimento humano está reconocido por el Código Alimentario Argentino (CAA), en el Artículo 682 (Resolución Conjunta SPReI N 261/2014 y SAGyP $\mathrm{N}^{\circ} 228 / 2014$ ), entendiéndose por quinua o quínoa a las semillas sanas, limpias y bien conservadas del género Chenopodium quínoa Willd. El principal uso de la quinoa está orientado a la alimentación humana, donde se utilizan las semillas enteras o procesadas de diferentes maneras.Sus 
granos son considerados uno de los más completos nutricionalmente [17]. Aparte de los macronutrientes, los estudios en quinoa están enfocados en sus compuestos bioactivos de naturaleza hidrofílica, como los compuestos fenólicos y flavonoides, que le otorgan capacidad antioxidante, con sus potenciales beneficios para la salud [28, 2,11, 26, 16, 22].

Dentro de los antioxidantes naturales encontrados en la quínoa, se destacan la importancia de los compuestos fenólicos, cuya aplicación en la formulación de potenciales alimentos funcionales hace interesante su extracción de manera selectiva. Los compuestos fenólicos, incluyendo los ácidos fenólicos, flavonoides y taninos, ejercen acciones preventivas y protectoras contra el cáncer, las alergias, los procesos inflamatorios y las enfermedades cardiovasculares $[10,15]$. Su empleo promueve el uso de antioxidantes naturales, que podrán reemplazar a los de origen sintético.

La extracción por solventes de compuestos bioactivos de materiales vegetales permeables, constituye una etapa importante en la elaboración de productos ricos en fitoquímicos. La aplicación de esta tecnología de bajo costo para obtener compuestos utilizados como aditivos o productos nutracéuticos, es una estrategia apropiada para la explotación de diferentes vegetales [8].

En consecuencia, el objetivo de este trabajo fue la optimización de las variables operativas del proceso de extracción de compuestos fenólicos bioactivos en quinoa: temperatura de secado del grano $(\mathrm{T})$, relación líquidosólido (L/S) y concentración de etanol en la solución extractiva $(\% \mathrm{Et})$.

\section{Materiales y Métodos}

La quínoa (Chenopodium quínoa Willd) fue provista por Quínoa Real, empresa que tiene sus cultivos en Yavi, Jujuy, Argentina (22 $\left.7^{\prime} 47^{\prime \prime} \mathrm{S} 65^{\circ} 27^{\prime} 44^{\prime \prime} \mathrm{O}\right)$. El acopio se realizó en el momento de la cosecha, en una cantidad tal que permitió asegurar el suministro necesariopara todos los ensayos con material proveniente de una sola partida, la que fue conservada en frascos de vidrio de $5 \mathrm{~L}$, con tapa hermética y al abrigo de la luz y el calor, hasta su empleo. Para la preparación de los extractos, se trabajó con quinoa previamente desaponificada por vía húmeda. Los granos se secaron en estufa (Neo Line con circulación de aire). El tiempo de secado quedó definido por una humedad final del 12\% (AOAC 15950.01), requerida para evitar el deterioro de los granos causado por microorganismos. Los granos de quinoadesaponificados y secos se molieron en molino IKA, hasta pasar por tamiz malla $\mathrm{n}^{\circ} 40$.

Para evaluar la posible interferencia de los lípidos presentes en la quinoa en la determinación de polifenoles mediante el método de Folin-Ciocalteau, se llevó a cabo un ensayo preliminar con una muestra de harina proveniente de granos desaponificados y secados a $60^{\circ} \mathrm{C}$, para la relación $\mathrm{L} / \mathrm{S} 20: 1$, variando el porcentaje de etanol en la mezcla hidroalcohólica entre el 0 y el 100\%.

\section{Diseño experimental y análisis estadístico}

La optimización de la extracción de los compuestos fenólicos se realizó sobre la base de un diseño experimental de 3 variables a 3 niveles, para lo cual se utilizó un diseño central compuesto centrado en las caras. Los parámetros y sus niveles fueron: temperatura de secado del grano(T): 40, 60 y $80^{\circ} \mathrm{C}$; relación solución extractiva Líquido/Sólido (L/S): 20:1, 30:1 y 40:1 y concentración de etanol en el solvente $(\% \mathrm{Et}): 30,50$ y $70 \% \mathrm{v} / \mathrm{v}$.

La Tabla 1 muestra los valores codificados de los tres niveles (variables) utilizados en el diseño experimental y su correspondiente valor real para los factores de entrada.

Tabla 1: Relación entre los valores codificados y valores reales de las variables independientes

\begin{tabular}{|c|c|c|c|}
\hline & $\mathbf{T}\left({ }^{\circ} \mathbf{C}\right)$ & \% Et & L/S \\
\hline Nivel -1 & 40 & 30 & 20 \\
\hline Nivel 0 & 60 & 50 & 30 \\
\hline Nivel +1 & 80 & 70 & 40 \\
\hline
\end{tabular}

La interacción lineal y los coeficientes cuadráticos se evaluaron mediante ANOVA $(p<0,05)$. Se determinaron los valores de F-test y probabilidad (p), para analizar la contribución estadística significativa de todos los términos. Los resultados del diseño experimental se procesaron mediante la aplicación de un análisis de regresión múltiple. El ajuste del modelo se verificó mediante el coeficiente de determinación $\left(\mathrm{R}^{2}\right)$ y la significancia de los términos del modelo se estableció con un nivel de confianza del 95\%. Las condiciones del proceso se optimizaron a través de los gráficos de superficie de respuesta. Todos los análisis se realizaron utilizando la versión de prueba gratuita del software Minitab 18 (Minitab, Pennsylvania, EE.UU).

\section{Obtención de extractos hidroalcohólicos}

Para la extracción de compuestos bioactivos, la harina obtenida por molienda de los granos secos se puso en contacto con la solución extractiva durante 1 hora en un agitador rotativo (Boeco Germany Orbital Shaker OS20 ), a $25^{\circ} \mathrm{C}$. Los extractos se filtraron y se preservaron a $4 \pm 1^{\circ} \mathrm{C}$ en recipientes color caramelo, hasta su posterior uso.

\section{Cuantificación de fenoles totales en los extractos de quinoa (CPT)}

Para la cuantificación del contenido de fenoles totales (CPT), se utilizó el método de Folin-Ciocalteu [25], basado en la oxidación de los compuestos polifenólicos a fenolatos en medio alcalino, formando un complejo de molibdeno-tungsteno de color azul. Se mezclaron 0,4 ml de muestra con $4 \mathrm{ml}$ de agua destilada, $0,4 \mathrm{ml}$ de reactivo de Folin-Ciocalteu y $2 \mathrm{ml}$ de solución saturada de carbonato de sodio. La mezcla se homogeneizó y se dejó reposar 2 
horas en oscuridad, hasta la medición de la absorbancia a $765 \mathrm{~nm}$ en espectrofotómetro (Shimadzu Spectrophotometer UV-1800). Las mediciones de absorbancia fueron realizadas por triplicado.

Los resultados fueron expresados como ácido gálico equivalente (AGE)/100 g de quinoa en base seca (b.s) utilizando una curva de calibración obtenida a partir de soluciones estándar de ácido gálico. La ecuación de la recta de calibración fue $\mathrm{Y}=0,0035 \mathrm{X}+0,0277$, con $\mathrm{R}^{2}=$ 0,998 en la que Y: absorbancia y X: concentración.

\section{Resultados y Discusión}

En la Figura 1 se muestranlos valores de CPT para harina desgrasada y sin desgrasar, proveniente de granos secados a $60^{\circ} \mathrm{C}$ y una relación L/S de 20:1.

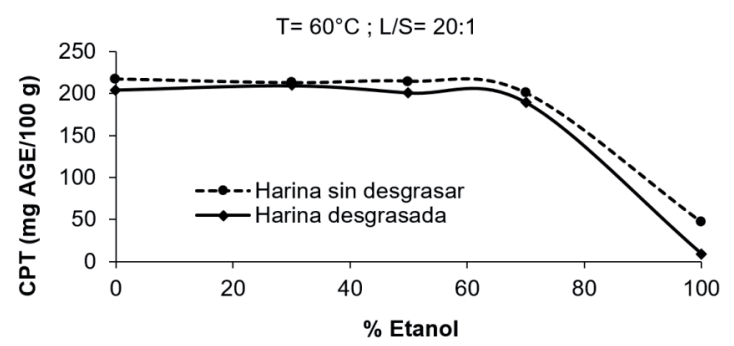

Figura 1: Contenido de polifenoles totales a partir de harina de quinoa previamente desgrasada y sin desgrasar (harina secada a $60^{\circ} \mathrm{C}$ y relación L/S empleada en la extracción 20:1)

No se encontraron diferencias significativas $(\mathrm{p}>0,05)$ entre los valores de CPT obtenidos a partir de harina desgrasada y sin desgrasar, para todo el rango de porcentajes de etanol de la solución extractiva.Considerando que el contenido lipídico de las semillas de quinoa no interfiere en la cuantificación de polifenoles por medio de la técnica de Folin-Ciocalteau, se prosiguió con los ensayos propuestos, trabajando sobre harina sin desgrasar.

En la Tabla 2 se observan los valores de CPT obtenidos para las diferentes condiciones de ensayo. Se requirieron 18 experiencias, agrupadas en 8 para verificar el modelo a dos niveles $(-1,1)$ para los 3 factores $\left(2^{\mathrm{k}}, \mathrm{k}=3\right)$; 4 réplicas en el punto central para evaluar el error puro y 6 ensayos restantes para testear los valores centrales de las caras del plan (puntos en estrella).

Los valores de CPT obtenidos coinciden con el intervalo informado por Abderrahim et al. [2], quienes encontraron rangos entre 128 a $452 \mathrm{mg} \mathrm{AGE/100} \mathrm{g} \mathrm{b.s.}$ Repo-Carrasco y Encina-Zelada [23], en cambio, informaron valores menores, comprendidos entre 35,29 a 139,94 mg AGE/ $100 \mathrm{~g}$ bs, cuando analizaron quince variedades de quinoas provenientes del Perú. Tang et al. [27] hallaron valores aún más bajos (46,7 a 68,2 mg AGE/ 100 g b.s.) para quinoas originarias de Canadá, al igual que Miranda et al [20], para seis ecotipos de quinoas chilenas (entre 14,22 y 65,53 mg AGE/ $100 \mathrm{~g}$ b.s).
Tabla 2: Valores respuesta del contenido de polifenoles totales

\begin{tabular}{|c|c|c|c|}
\hline $\begin{array}{l}\mathrm{T} \\
{ }^{\circ} \mathrm{C}\end{array}$ & $\%$ Et & L/S & $\begin{array}{c}\text { CPT } \\
\text { mg AGE/100 g quinoa }\end{array}$ \\
\hline 40 & 30 & 20 & $214 \pm 1$ \\
\hline 80 & 30 & 20 & $191 \pm 4$ \\
\hline 40 & 70 & 20 & $182 \pm 10$ \\
\hline 80 & 70 & 20 & $179 \pm 5$ \\
\hline 40 & 30 & 40 & $209 \pm 4$ \\
\hline 80 & 30 & 40 & $204 \pm 9$ \\
\hline 40 & 70 & 40 & $185 \pm 4$ \\
\hline 80 & 70 & 40 & $183 \pm 9$ \\
\hline 60 & 50 & 30 & $233 \pm 2$ \\
\hline 60 & 50 & 30 & $223 \pm 2$ \\
\hline 60 & 50 & 30 & $227 \pm 1$ \\
\hline 60 & 50 & 30 & $229 \pm 3$ \\
\hline 40 & 50 & 30 & $214 \pm 5$ \\
\hline 80 & 50 & 30 & $203 \pm 12$ \\
\hline 60 & 30 & 30 & $218 \pm 4$ \\
\hline 60 & 70 & 30 & $191 \pm 1$ \\
\hline 60 & 50 & 20 & $190 \pm 10$ \\
\hline 60 & 50 & 40 & $202 \pm 6$ \\
\hline
\end{tabular}

El perfil de compuestos fenólicos en las plantas es muy diverso, dependiendo de la especie vegetal, la variedad, la parte de la planta considerada (semilla, fruto, tallo, hojas, etc.), las condiciones agroclimáticas de cultivo, el grado de maduración, como así también de aspectos tecnológicos relacionados con el procesamiento y almacenamiento, entre otros [21].

En la Tabla 3 se muestra el análisis estadístico de los resultados. Se observa que la temperatura y el porcentaje de etanol presentaron un efecto significativo $(\mathrm{p}<0,05)$ en el contenido de fenoles totales, como así también las interacciones cuadráticas de temperatura, porcentaje de etanol y relación L/S. La variable de mayor influencia en la extracción de CPT fue el porcentaje de alcohol en el solvente.

Tabla 3: Resultados del ANOVA correspondiente al contenido de polifenoles totales en extractos de semilla de quinoa, obtenidos bajo diferentes condiciones de $\mathrm{T}$ de secado $\left(\mathrm{T}^{\circ} \mathrm{C}\right)$, porcentaje de etanol en el solvente (\%Et) y relación L/S.

\begin{tabular}{|c|c|c|c|c|c|}
\hline $\begin{array}{l}\text { Fuente de } \\
\text { variación }\end{array}$ & $\begin{array}{l}\text { Grados de } \\
\text { libertad }\end{array}$ & $\begin{array}{c}\text { Suma de } \\
\text { cuadrados }\end{array}$ & $\begin{array}{c}\text { Cuadrado } \\
\text { medio }\end{array}$ & Razón-F & Valor-P \\
\hline $\mathrm{T}$ & 1 & 193,60 & 193,60 & 9,06 & 0,013 \\
\hline$\%$ Et & 1 & 1345,60 & 1345,60 & 62,94 & 0,000 \\
\hline $\mathrm{L} / \mathrm{S}$ & 1 & 72,90 & 72,90 & 3,41 & 0,095 \\
\hline $\mathrm{T}\left({ }^{\circ} \mathrm{C}\right)^{*} \mathrm{~T}\left({ }^{\circ} \mathrm{C}\right)$ & 1 & 126,04 & 126,04 & 5,90 & 0,036 \\
\hline$\% \mathrm{Et} * \% \mathrm{Et}$ & 1 & 315,37 & 315,37 & 14,75 & 0,003 \\
\hline$L / S * L / S$ & 1 & 1001,04 & 1001,04 & 46,83 & 0,000 \\
\hline Modelo & 7 & 5480,72 & 782,96 & 36,63 & 0,000 \\
\hline \multirow{2}{*}{\multicolumn{3}{|c|}{$\begin{array}{c}\text { Resumen del modelo } \\
4,62358 \\
93,62 \%\end{array}$}} & S & $\mathrm{R}^{2}$ & $\begin{array}{c}\mathrm{R}^{2} \\
\text { (ajustado) }\end{array}$ \\
\hline & & & $87,46 \%$ & & \\
\hline
\end{tabular}

A partir del análisis de regresión múltiple, se obtuvo la siguiente ecuación polinómica de segundo orden, con $\mathrm{p}<$ 0,05 y $\mathrm{R}^{2}=93,62 \%$ : 
CPT (mg AGE/100g quinoa b.s. $)=-47,2+1,843 \mathrm{~T}\left({ }^{\circ} \mathrm{C}\right)$ $+2,139 \% \mathrm{Et}+11,89 \mathrm{~L} / \mathrm{S}-0,01719 \mathrm{~T}\left({ }^{\circ} \mathrm{C}\right) * \mathrm{~T}\left({ }^{\circ} \mathrm{C}\right)$ - 0,02719\%Et*\%Et - 0,1937 L/S*L/S

En la Figura 2 se muestran los valores de CPT experimentales y teóricos estimados por el modelo, observándose el grado de coincidencia entre los mismos.

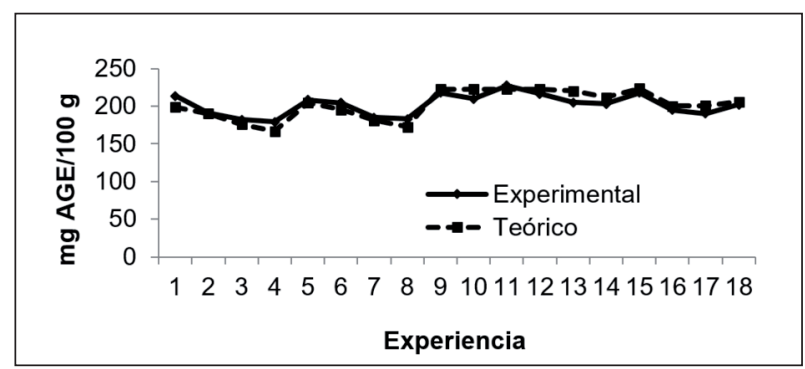

Figura 2: Valores del contenido de polifenoles totales experimentales y predichos por el modelo

Las superficies de respuesta de la Figura 3, muestran el comportamiento del contenido de polifenoles totales obtenido en función de las 3 variables estudiadas. Fijando la relación L/S se observa que el CPT aumenta para valores de temperatura próximos a $60^{\circ} \mathrm{C}$ donde se obtiene el máximo contenido de polifenoles totales. Manteniendo constante la temperatura, el contenido de polifenoles aumenta cuando la concentración de alcohol en el solvente es próxima al $40 \%$. Por último, para una mezcla extractiva de concentración de etanol fija, se logra un máximo del CPT cuando la relación L/S es de 30:1, decreciendo para relaciones de 20:1 y 40:1.

Figura 3: Superficie respuesta para el contenido de compuestos fenólicos totales (CPT) en función de las variables de proceso: temperatura (T), \%Etanol (\%Et) y relación líquido de extracción/sólido (L/S).

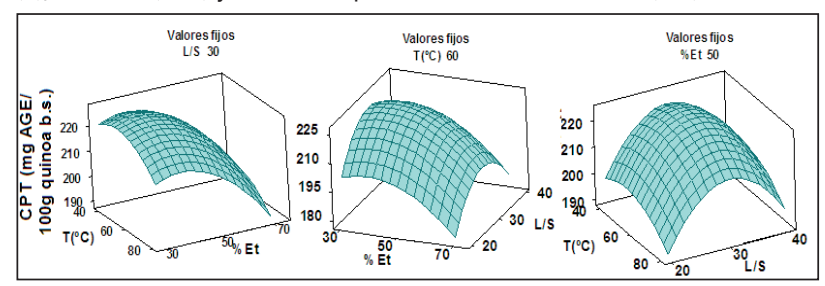

En la Figura 4 se presentan las variables del proceso que optimizaron la respuesta del contenido de polifenoles totales. Las mismas fueron: temperatura de secado del grano de $54^{\circ} \mathrm{C}$, concentración de etanol en la mezcla extractiva de 39\% y relación liquido/sólido de 31:1.

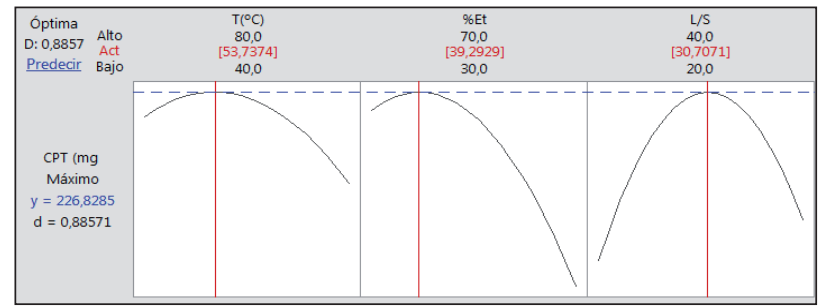

Figura 4: Optimización de la respuesta del contenido de polifenoles totales
Miranda et al. (2010), evaluaron el impacto de diferentes temperaturas de secado en las propiedades nutricionales, contenido de compuestos fenólicos totales y capacidad antioxidante en granos de quinoas, encontrando que las temperaturas de secado entre 60 y $80^{\circ} \mathrm{C}$ resultan en una notable degradación de los compuestos fenólicos totales, aunque no evidencian pérdidas significativas con respecto a la capacidad antioxidante. Vidaurre-Ruiz et al. (2017) encontraron que si bien después del proceso de secado algunos compuestos bioactivos de las variedades de quinoa Negra Collana y Pasankalla, disminuyen significativamente, se pueden formar otros compuestos con mayor capacidad antioxidante.

Para la relación L/S, el máximo de CPT se registró para una relación $\mathrm{L} / \mathrm{S}$ de 31:1. La relación óptima de $\mathrm{L} / \mathrm{S}$ a emplear en la extracción dependerá de dos consideraciones fundamentales: la cantidad máxima conveniente de solvente en la práctica, está limitada por las consecuencias relacionadas con la dilución que se produce de los sustratos de interés, al utilizar mayores volúmenes de solvente [18]. Por otro lado, el rendimiento del extracto en compuestos de interés está directamente relacionado con la cantidad de material sólido de partida, por lo que el valor óptimo resultará principalmente del compromiso de éstas dos consideraciones [7].

En el caso del solvente, se observó un aumento en el CPT para mezclas extractoras de $39 \%$ de porcentaje de etanol lo que indicaría la presencia mayoritaria de compuestos bioactivos de naturaleza hidrofílica en la quinoa $[28,2,11$, 26, 16, 22, 24].

Químicamente, los compuestos fenólicos son sustancias químicas que poseen un anillo aromático, con uno o más grupos hidroxilos incluyendo derivados funcionales. La naturaleza de los polifenoles varía desde moléculas simples, como los ácidos fenólicos hasta compuestos altamente polimerizados, como los taninos. En las plantas, se presentan en forma conjugada con uno o más residuos de azúcar unidos a los grupos hidroxilos, aunque en algunos casos se pueden producir uniones directas entre una molécula de azúcar y un carbono aromático. Por ello la forma más común de encontrarlos en la naturaleza es en forma de glicósidos, que son solubles en agua y en solventes orgánicos. Se ha reportado el uso de etanol, metanol y acetona $[30,9,5]$ y sus mezclas con agua en diferentes proporciones. En particular, las mezclas de agua y etanol son las más utilizadas dado su eficiencia, selectividad, bajo costo, baja toxicidad y facilidad de eliminación [31,14]. Al modificar las proporciones de agua y etanol, varía la polaridad del solvente y como consecuencia se consigue cambiar la naturaleza del extracto obtenido, en función de la solubilidad de los diferentes compuestos fenólicos presentes en la matriz vegetal [12].

Sin embargo, no existe un método y solvente definidos, pues ello dependerá de la naturaleza química de los compuestos a extraer, de la cantidad y posición de sus 
grupos hidroxilo, del tamaño molecular, así como de la concentración del solvente, temperatura, tiempo de contacto, tamaño de partícula y relación masa-solvente, entre otros $[3,4,13,7]$.

\section{Conclusiones}

En este estudio preliminar establecieron valores óptimos para la temperatura, el porcentaje de etanol en el solvente y la relación $\mathrm{L} / \mathrm{S}$, para el proceso de extracción de compuestos fenólicos a partir de quinoa (Chenopodium quínoa Willd). Será oportuno continuar los estudios con otras variedades de quinoa o modificando las temperaturas de extracción.

\section{Referencias}

1. Abugoch, L.E. 2009. Quinoa (Chenopodium quinoa Willd): composition, chemistry, nutritional and functional properties. Advances in Food and Nutrition Research, 58, $1-31$.

2. Abderrahim, F.; Huanatico, E.; Segura, R., Arribas, S.; Gonzales, M.; Condezo-Hoyos, L. 2015. Physical features, phenolic compounds, betalains and total antioxidant capacity of coloured quinoa seeds (Chenopodium quinoa Willd) from Peruvian Altiplano. FoodChemistry, 183, 83-90.

3. Amyrgialaki, E.; Makris, D. P.; Mauromoustakos, A.; Kefalas, P. 2014. Optimisation of the extraction of pomegranate (Punicagranatum) husk phenolics using water/ethanol solvent systems and response surface methodology. Industrial Crops and Products 59:216-222.

4. Aspé, E.; Fernández, K. 2011. Comparison of phenolic extracts obtained of Pinusradiata bark from pulp and paper industry and sawmills industry. Maderas. Ciencia y Tecnología, 13(3), 243-252.

5. Boeing, J.S.; Oliveira Barizão, E.; Costa e Silva, B.; Fernandes Montanher, P.; de Cinque Almeida, V.; Vergilio Visentainer, J. 2014. Evaluation of solvent effect on the extraction of phenolic compounds and antioxidant capacities from the berries: application of principal component analysis. Chemistry Central Journal, 8, 48-57.

6. Capriotti, A.L.; Cavaliere, C.; Crescenzi, C.; Foglia, P.; Nescatelli, R.; Samperi, R.; Lagana, A. 2014. Comparison of extraction methods for the identification and quantification of polyphenols in virgin olive oil by ultra-HPLC-QToF mass spectrometry. Wood Chemistry 158, 392-400.

7. Carciochi, R. A. 2014. Obtención de ingredientes alimenticios con capacidad antioxidante mejorada por aplicación de distintos procesos a semillas de quinoa (Chenopodium quinoa). Tesis Doctoral de la Facultad de Farmacia y Bioquímica de la Universidad de Buenos Aires, Argentina.

8. Da Silva Campelo Borges, G.; GracieliKunradi Vieira, F.; Copetti ,C.; Valdemiro Gonzaga, L.;Fett R. 2011. Optimization of the extraction of flavanols and anthocyanins from the fruit pulp of Euterpeedulis using the response surface methodology. Food Research International, 44, 708715.

9. Dent, M.; Dragovic-Uzelac, V.; Penic, M.; Brncic, M.; Bosiljkov, T.; Levaj, B. 2013. The effect of extraction solvents, temperature and time on the composition and mass fraction of polyphenols in Dalmatian Wild Sage (Salvia officinalis L.) extracts. Food Technology and Biotechnology, 51, 84-91.

10. Dini, I, Tenore, G. C and Dini, A. 2010. Antioxidant compound contents and antioxidant activity before and after cooking in sweet and bitter Chenopodium quinoa seeds. LWT-Food Sience and Technology, 43, 447-451.

11. Fisher, S; Wilckens, R; Jara, J; Aranda, M. 2013. Variation in antioxidant capacity of quinoa (Chenopodium quinoa Will) subjected to drought stress. Industrial Crops and Products, 46, 341- 349.

12. Galvan d'Alessandro, L.; Kriaa, K.; Nikov, I.; Dimitrov, K. 2012. Ultrasound assisted extraction of polyphenols from black chokeberry. Separation and Purification Technology, 93, 42-47.

13. Gironi, F.; Piemonte, v. 2011. Temperature and solvent effects on polyphenol extraction process from chestnut tree wood. Chemical Engineering Research and Design, 89, 857-862.

14. Gong, Y.; Hou, Z.; Gao, Y.; Xue, Y.; Liu, X.; Liu, G. 2012. Optimization of extraction parameters of bioactive components from deffated marigold (Tageteserecta L.) residue using response surface methodology. Foods and Bioproducts Processing, 90, 9-16.

15. Han, X.; Shen, T.; Lou, H. 2007. Dietary polyphenols and their biological significance. International Journal of Molecular Sciences, 8, 950-988.

16. Hirose, Y.; Fujita, T.; Ishii, T.; Ueno, N. 2010. Antioxidative properties and flavonoid composition of Chenopodium quinoa seeds cultivated in Japan. Food Chemistry, 119, 1300-1306.

17. López, L.; Capparelli, A. \& Nielsen, A. 2011. Traditional post-harve processing to make quinoa grains (Chenopodium quinoa var. quinoa) apt for consumption in Northern Lipez (Potosi, Bolivia): ethnoarchaeological and archaeobotanical analyses. Archaeological and Anthropological Sciences, 3, 49-70.

18. Mafart, P.; Béliard, E. 1992. Génie Industrial Alimentaire. Tomo II. Techniques Séparatives. Editions Tec \& Doc, Lavoisier.

19. Miranda, M.; Vega-gálvez, A.; Martínez, E.; López, J.; Marín, R.; Aranda, M.; Fuentes, F. 2013. Influence of contrasting environments on seed composition of two quinoa genotypes: nutritional and functional properties. Chilean Journal of Agricultural Research, 73(2), 108-116.

20. Miranda, M.; Vega-Gálvez, A.; López, J.; Parada, G.; Sanders, M.; Aranda, M. et al. 2010. Impact of air-drying temperature on nutritional properties, total phenolic content and antioxidant capacity of quinoa seeds (Chenopodium 
quinoa Willd). Industrial Crops and Products. 32, 258-263.

21. Naczk, M.; Shahidi, F. 2006. Phenolics in cereals, fruits and vegetables: Occurrence, extraction and analysis. Journal of Pharmaceutical and Biomedical Analysis, 41, 1523-1542.

22. Repo-Carrasco-Valencia, R.; Hellström, J. K.; Pihlava, J.; Mattila, P. 2010. Flavonoids and other phenolic compounds in Andean indigenous grains: Quinoa (Chenopodium quinoa), kañiwa (Chenopodium pallidicaule) and kiwicha (Amaranthus caudatus). FoodChemistry, 120, 128-133.

23. Repo-Carrasco, R.; Encina Zelada, CR. 2008. Determinación de la capacidad antioxidante y compuestos fenólicos de cereales andinos: Quinua (Chenopodium quinoa), Kañiwa (Chenopodium pallidicaule) y Kiwicha (Amaranthus caudatus). Revista Sociedad Química del Perú. 74(2), 85-99.

24. Silva, E. M.; Rogez, H.; Larondelle, Y. 2007. Optimization of extraction of phenolics from Inga edulis leaves using response surface methodology. Separation and Purification Technology, 55, 381-387.

25. Singleton, V. L.\& Rossi, J. A. 1999. Colorimetry of total phenolics with phosphomolybdic-phosphotungstic acid reagents. American Journal of Enology and Viticulture, 16(3), 144-158.
26. Stikic, R.; Glamoclija, D.; Demin, M.; Vucelic-Radovic, B.; Jovanovic, Z.; Milojkovic-opsenica, D.; Jacobsen, S.; Milovanovic, M. 2012. Agronomical and nutritional evaluation of quinoa seeds (Chenopodium quinoa Willd) as an ingredient in bread formulations. Journal of Cereal Science, 55, 132-138.

27. Tang, Y.; Li, X.; Zhang, B.; Chen, P. X.; Liu, R.; Tsao, R. 2015. Characterisation of phenolics, betanins and antioxidant activities in seeds of three Chenopodium quinoa Willd genotypes. Food Chemistry, 166, 380-388.

28. Valencia, Z.; Cámara, F.; Ccapa, K.; Catacora, P.; Quispe, F. 2017. Compuestos bioactivos y actividad antioxidante de Semillas de quinua peruana (ChenopodiumquinoaWilld). Sociedad Químerú, 83(1), 16- 29.

29. Vidaurre-Ruiz, J. M; Días-Rojas, G, Mendoza-Llamo, E, SolanoCornejo, M. A. (2017). Variación del contenido de Betalaínas, compuestos fenólicos y capacidad antioxidante durante el procesamiento de la quinua (Chenopodium quinoa $W$.). Revista de la Sociedad Química del Perú, 83(3), 319-330.

30. Wang, Z.; Pan, Z.; Ma, H. Atungulu; G. G. 2011. Extract of phenolics from pomegranate peels. The Open Food Science Journal, 5, 17-25.

31. Zhang, G.; He, L.; Hu, M. 2011. Optimized ultrasonic-assisted extraction of flavonoids from Prunella vulgaris L. and evaluation of antioxidant activities in vitro. Innovative Food Science and Emerging Technologies, 12, 18-25. 